\title{
Anger: Examining Anger Variations and their Effects
}

\author{
Anil Thomas \\ NLP Trainer \& Gestalt Master \\ Mumbai, India
}

\author{
Pranjali Vatsa \\ Graduate from Sophia College \\ Mumbai, India
}

\begin{abstract}
Anger is one of the simplest and primary emotions felt by human beings but this emotion can single handedly give rise to various negative emotions like frustration, aggression, irritation, sadness, grief and pain. Anger, being the root cause of all these negative emotions, makes a very interesting topic to study. Here, we have tried to look into anger and its variations that can be expressed in the form of several emotions like mild irritation, annoyance, reduced tolerance level, frustration, full blown tantrum etc.

One hundred and eighty two $(\mathrm{N}=182)$ individuals were studied from the Indian population belonging to different religions and sects. This data was collected via descriptive google forms. The Novaco Anger Scale (NAS) was used as the questionnaire. From this sample, we concluded that majority of the responders ranked towards the lower half of the anger scale regardless of age, sex and religion they belonged to.
\end{abstract}

Keywords: Anger, Tolerance, Bullying, Rage, Aggression

\section{Introduction}

In simple terms, anger is an emotion which predominantly occurs due to the failure of achieving a particular thing, task or goal. Human beings are spontaneous and impulsive creatures.

This Research Paper is on 'Anger and it's Expressions' and has been authored by Anil Thomas

This paper has been co-authored by Pranjali Vatsa, an Intern part of the Global Internship Research Program (GIRP) who graduated from Sophia College, Mumbai India.

Kainaz Bharucha served as a Statistical Research Adviser who is an Intern part of the Global Internship Research Program (GIRP). This paper has been critically reviewed and proofread by Shaifila Ladhani.

We thank our Trupti Machwe (Our Editor in Chief) and Priya Pawar,(Deputy Editor in Chief) for their dedicated time and contribution towards IJNGP.

Correspondence concerning this article should be addressed to

E-mail: office@ijngp
Wants never end but wants aren't always achieved. If an individual wants something and is unable to attain it, a specific sort of sensation is felt. This specific sort of sensation is often described as an irritation or a churning energy or simply as discomfort.

Anger is linked to the sympathetic nervous system's "fight, flight, or fright" response, which prepares humans to battle. Fighting, on the other hand, does not always allude to throwing punches. It may inspire communities to take action against injustice by altering laws or establishing new norms. "A strong sensation of violence, discontent, or hostility" is commonly used to define anger. It's crucial to remember that rage is a natural and universal human emotion. There are a variety of situations and occurrences in life that might make someone angry.

Supported by Devashish Polymers

Research and Learning Grants' Partner 
Anger usually develops when a person feels threatened, upset, or otherwise disturbed from a serene emotional state. Anger can also be mixed with other emotions.

Anger is essentially an effective response to stressful situations and events of life. It is biological, psychological and social in nature with major links to survival and preservation mechanisms of life. Threat perception is inextricably linked to its activation, and symbolic structures govern it. Anger can be prolonged or disengaged by cognitive processing of anger-provoking experiences. It is primed and defined by neurophysiological arousal, and as a high arousal state, it can operate as an internal stressor, producing wear and tear on the body when engaged repeatedly. Anger is linked to approaching motivational systems in the brain and can lead to violent conduct. While social standards limit rage expression, it can be a part of an adversarial manner of coping with daily stressors, particularly when dealing with interpersonal conflict. Anger's position as a catalyst for violent behaviour is both interpersonal and societally detrimental. Through transdiagnostic processes like selective attention, threat perception, and rumination, anger dysregulation causes impairment in functioning across life domains and is linked to a variety of mental diseases. For a wide range of clinical populations, the efficacy of psychotherapy therapies for anger, particularly cognitivebehavioural therapy, is well-established.

Shot suggested that in order to feel emotion, people must first experience physiological arousal, which is subsequently labelled as emotion. Emotion words that have been culturally defined and offered are employed in this procedure. Ways of emotion expression, face expression and recognition of emotions, nature of commonly experienced emotions, and affect appraisal are some more examples of emotional elements with cultural diversity. There have been evidence and instances that indicate a positive relationship between unpleasant emotions and illnesses such as atherosclerosis of coronary heart disease, according to Goldel Hill et al. (2006). (CHD). The HPA axis and sympathetic nervous system, which are activated by anger, can have a direct impact on cardiovascular disease by causing an excessive release of corticosteroids and catecholamine. The release of such stress hormones can trigger a cascade of events, including hemodynamic and metabolic changes, vascular issues, and heart rhythm disturbances. Anger can also encourage the adoption of a lifestyle that proves to be unhealthy for an individual (smoking, intake of high caloric aliments, alcohol and caffeine consumption).

Rage can be expressed in at least three ways: it can be driven by constructive reasons (to solve a problem), or it can be motivated by deconstructive reasons (to justify someone's sentiments or to deepen their state of anger). As a result, some research found that anger increases the risk of coronary heart disease, while others indicated that in particular instances, rage manifestation can be protective.

The goal of a study published in 2010 in Canada was to uncover health hazards connected with the three forms of rage manifestations. The individuals were tracked for ten years, and the findings demonstrated that decreased constructive anger in men and greater destructive anger rationalisation in both men and women was linked to a higher risk of a CHD incidence in ten years. In women who had already had a coronary event, outward expression and the repression of anger appear to be linked to prognosis.

Threatening people are presented to pessimistic mentalities and are questioned by others, according to Vella and Friedman (2009), which might lead to frequent rage experimenting and other associated behaviours. Situations that require anger inhibition may be more common in hostile people's daily lives than interactions that allow them to express their wrath. When compared to people who express their anger freely, suppressing anger has been associated with increased carotid artery stiffness and intima-medial thickness, both of which are subclinical markers of coronary heart disease. According to some data, hostile people who suppress their anger are more likely to develop substantial coronary atherosclerosis than hostile people who express their rage.

\section{Cultural Variation in Anger}

Willard Gaylin, MD argued in his book "The Rage Within: Anger in Modern Life", the bestseller of the year 1984, that in the American lifestyle anger and rage are two of the most prevalent emotions felt by people. Threats, 
verbal abuse, litigation, and public aggression are all too common, according to him, and he concludes, "We are allowing more brutality than is prudent and legitimising more greed than is safe." Emotions are physiologically based and genetically encoded, and they are controlled by the amygdala, a gland in the limbic part of the brain. Internal sensations such as love, sorrow, fear, and rage are also manufactured locally, culturally, and contextually. As a result, culture shapes and is formed by emotions. Furthermore, some emotions are valued and revered in every society, while others are viewed as harmful and disruptive. Anger, fear, grief, and thankfulness may be encouraged or discouraged in a culture. The moral interests, values, judgments, and visions of a culture will be reflected in the extent to which it fosters or discourages an emotion. We know about a person's feelings through language since each person's feelings are internalised. People tell us if they are happy or sad. The study of emotions necessitates the use of language. Emotional words must be carefully understood both inside a cultural group and between civilizations. Anger is an emotion that not everyone in a cultural group shares equally. Intensity levels may be determined by gender or social class. The more extreme types of rage include the threat of violence and a desire for vengeance. People have been known to behave out of such extreme rage that they lose awareness and psychologically black out. If culture shapes one's view of anger, it's useful to look at how other civilizations have expressed anger to see whether it's tolerated and promoted or discouraged and restricted. We can learn about a wide range of thoughts on anger in this way, thanks to comparative analysis. For example, anger in Malay means outraged, the Ifaluk term for anger means righteous indignation, and the English definition of anger according to Webster's Dictionary is: a strong sense of discontent, hostility, indignation, or impatience. As a result, rage in English suggests that someone else is to blame, as well as strong feelings of good and evil, right and wrong. Anger was seen as a cause of disruption and disturbance by early Greek and Roman dramatists, historians, poets, and philosophers. Author William Harris just published a book titled "Restraining Rage: the Ideology of Anger Control in Classical Antiquity," which examines ancient classical writers' beliefs on wrath control and why they supported restraint or even removal of anger. Homer and Hesiod were the first to write on anger management. These storytellers were frequently confronted with the negative consequences of rage. "Wrath - Goddess sang the wrath of Peleus' son Achilles," begins the Iliad, "that cost the Achaeans innumerable losses, throwing down to the House of Death so many brave souls." Homer discusses rage throughout the epic since both gods and humanity are prone to it. When he puts these words of regret into Hector's mouth, he counsels moderation of anger: "I wish strife would disappear from among Gods and men and also anger, which makes even wise men get into an evil temper. I'll say that Agamemnon, King of men, made me angry. However, what is done is better left alone, though we resent it still, and we must by force curb the dear passion in our breast." The conversation regarding anger restraint became more intense, heated, and elaborated as time went on. Anger, according to historians Herodotus and Thucydides, impairs reason and destroys relationships. Both placed a high emphasis on leaders and citizens who were able to control their fury. Anger was considered a "soul illness" by Plato and Aristotle. Aristotle believed that "those who do not display righteous wrath are considered as fools," but that "those who do not show righteous anger in the right way at the right moment and to the right person are viewed as fools." Anger was discussed by Epicureans and Stoics alike. Epicureans were moderates who believed that excessive anger was bad, but that some fury was necessary to get things moving. Epicureans were moderates who believed that excessive anger was harmful, but that some anger was necessary to arouse passion and motivation. Anger absolutists, as the Stoics were known, were adamantly opposed to it. They believed that all emotions should be subordinated to logic. "Anger as a desire for avenging injury cost the human race more than any plague" wrote Roman writer Seneca. "To govern one's mind and speech when angry is a mark of a great ability" Cicero observed. There were several references to rage in both the Old and New Testaments. Moses was consumed with many forms of passion - indignation, wrath, fury, and rage - to the point where he was unable to enter the Promised Land. When Jesus overturned the money-changers who were plundering the poor, he showed his indignation. In general, however, the Bible states that "anger rests in the bosom of a fool" and that "do not let 
the sun go down on your anger." As Christians developed their understandings of anger, it became connected with righteous indignation, which might be expressed in certain circumstances, but wrath and rage, which could lead to violence and a desire for retribution, were rejected. There has been significant ambiguity concerning emotions, keeping in mind that tolerance of rage varies from era to era and culture to culture within the general Judeo-Christian legacy. Scholars considered reason to be superior to emotions, thinking to be superior to feeling, and dispassion to be superior to passion. People were supposed to be at the mercy of their emotions, or to be "eaten away" or "blinded" by emotions. Emotions were considered questionable since they couldn't be controlled. The western worldview is built on these dichotomies, preconceptions, and biases.

According to traditional Indian philosophy, emotions are born out of desires. Desire was mainly seen in a negative light as it emphasizes that an individual needs something, that something is incomplete. This negates the Indian concept of having everything within oneself. A craving when not satisfied or upset leads to outrage (Krodha), envy (Asuya), despondency (Dukha), and languishing. Want when satisfied prompts bliss (Sukha) and happiness. Indian sacred texts caution that satisfaction of a craving would be able and most frequently prompts eagerness (Lobha). One desires for increasingly more of it. There is a longing for bigger belonging and more prominent delight. These when engaged, one might foster pomposity (Mada) and jealousy (Matsarya). There is an extra passionate encounter of dread (Bhaya) of losing anything that one has. These feelings cloud one's scholarly separation and lead one to passionate and mental aggravation.

Lu and Gilmour did a cross-cultural study on happiness and discovered that the American conception of happiness prioritised upbeatness, whereas the Chinese conception of happiness prioritised solemnity and reserve. Uchida and Kitayama discovered that Japanese people associate happiness with low arousal good emotions, but Americans associate happiness with high arousal positive emotions.

Kacen and Lee compared Caucasians and Asians in a cross-cultural study. The researchers employed a four-item bipolar arousal scale, which comprises emotion descriptors that represent distinct arousal levels. The arousal scale's emotion items were stimulated-relaxed (reversed), calm-excited (reversed), frenzied-sluggish (reversed), and unaroused-aroused. The findings revealed that Caucasians were more likely than Asians to be in high arousal emotional states (i.e., stimulated, enthusiastic, frantic, and aroused), while Asians were more likely to be in low arousal emotional states (i.e., calm, relaxed, and relaxed) (i.e., relaxed, calm, sluggish, and unaroused). Furthermore, Tsai and colleagues found that the higher the individuals' cardiovascular arousal level during interpersonal tasks, the closer they were to an American rather than a Chinese cultural orientation.

Cultural differences in physiological and behavioural elements of emotion can also be identified. Scherer et al discovered that Japanese individuals reported considerably fewer physiological symptoms than their American and European counterparts. One probable explanation, according to Mesquita and Frijda, is that their physiological reactions to emotions differ. Furthermore, the actions associated with different levels of emotional arousal vary by culture. To produce high arousal emotions, Westerners tend to participate in more active sports than Easterners. Furthermore, parents encourage their children to participate in activities that are likely to evoke culturally desirable feelings. Western women, for example, are said to encourage their children to play games that raise their emotional arousal level. As a result, cultural disparities in emotional arousal level manifest themselves at a young age.

\section{Causes of Anger}

We feel angry when we think that we are being treated unfairly. This easily comes in our way of achieving our goals and achieving a content state. The cognitive behaviour theory says anger is caused by a number of causes, including:

1. Past experiences

2. Learned behaviours

3. Genetic makeup and predispositions

4. Absence of problem solving ability

Dr. Harry Mills claims that rage is a taught emotion rather than one that we are born with (2005). We learn to feel furious in a variety of ways. We learn as youngsters by imitating the 
behaviour of others around us. Getting born and spending years growing up in an environment where arguments and raised voices are day to day occurrences, for example, can teach a youngster that such conduct is normal and that punishing people without cause is okay. The youngster may grow up without realising he or she has an anger problem. These children may develop violent and antagonistic attitudes toward their peers and others as they grow older. This acquired behaviour has the potential to turn a child into a bully. Bullying is defined as a pattern of hostile behaviour that is repeated over time. Bullies act combatively and truculently because it gives them a surging feeling of absolute power over other individuals. When they mistreat someone, they discover that others feel respect for them or are scared of them as a result of their aggressive actions. Because they have learnt that their activities make them popular, the youngster tends to grow more aggressive in their behaviour. Bullying victims, ironically, learn to be angry as a result of their constant exposure to aggressive and injurious behaviour. Their rage and need for vengeance grows, prompting them to develop anger disorders of their own. They grow enraged and seek vengeance not only on the person who mistreated them, but also on others. The bully now becomes the victim. Bullying isn't just a problem for youngsters and teenagers. Bullying affects adults as well. It might happen at home, at school, or in the office. Adults who are angry will lash out at their close family, friends, coworkers, and even complete strangers. They vent their rage on others, hoping that someone else would suffer the emotional damage and trauma they have endured; they want to see someone else experience the anguish, whether it is physical, mental, or emotional.

Social learning hypothesis, proposed by Albert Bandura, underlines the significance of noticing, demonstrating, and emulating the practices, perspectives, and enthusiastic responses of others. Kids notice individuals around them acting in different ways. This is delineated during the popular Bobo doll experiment done by Bandura in the 1960s. The investigation included presenting kids to two distinct grown-up models; a forceful model and a non-forceful one. In the wake of seeing the grown-up's conduct, the youngsters would then be set in a room without the model and were seen to check whether they would emulate the practices they had seen before. As indicated by Bandura, the vicious conduct of the grown-up models toward the dolls persuaded youngsters to think that such activities were satisfactory. He likewise recommended that accordingly, kids might be more disposed to react to disappointment with hostility later on.. .According to Loo (2005), an experienced negotiator and an expert in conflict resolution, are the two main causes of rage: an internal source and an external source. Anger is a result of an internal source. Four types of thought processes have been pointed out by psychologists that lead to anger internally:

1. Emotional reasoning: Some people tend to perceive things in life from an emotional perspective. This leads them to misconclude or misinterpret ordinary situations and events. They tend to get irritated very quickly and always think in a the-world-vs-me outlook.

2. Low frustration tolerance: Everyone possesses a low tolerance for annoyance at some stage in their lives. Our tolerance for annoyance deteriorates due to stress related anxiety further causing us to perceive ordinary things as threats to our person.

3. Unreasonable expectations: Some people tend to expect unreasonable things from situations without taking a minute to understand them. When things don't go their way, it results in either a tantrum or a sort of an episode. Rage, restlessness and aggression is seen as the individual's tolerance level diminishes.

4. People-rating: Some people tend to judge and categorise people into certain slots. This labelling and stereotyping reduces others to mere tags leading them to dehumanise and become unemotional towards others' feelings.

Individual's level of tolerance for frustration reduces significantly because of environmental factors in their lives. It is plain to see that low resilience levels of dissatisfaction factor into both inside and outer wellsprings of outrage. Perceiving these elements might assist us with managing our annoyance and assist with settling our displeasure issues. The accompanying four factors that we manage consistently, which cause our dissatisfaction resistance levels to diminish are: 
1. Stress/Anxiety

2. Physical and emotional pain

3. Substance abuse and alcoholism

4. Quick irritations like "having a bad day"

We deal with stress and worry on a daily basis, whether at work, at home, at school, or on the way home. Our stress levels rise, which can lead to a reduction in our tolerance for annoyance. Adults, teenagers, and children are all affected. Workplace and school-related stress frequently spills over into our homes, affecting our families. Even the commute home might cause an increase in tension and anxiety levels. You return home and your companion has many grumblings about the kids not having any desire to get their work done, their consistent battling, which can make you shout and make absolutely crazy. You've completely let go over your capacity to endure disappointment. What has happened is that you have joined your expert disappointments and drive with homegrown worries. This type of stress and anxiety is linked to an increase in domestic violence and child abuse. We have a far lower tolerance for impatience when we are in bodily or mental discomfort. Our discomfort becomes the focus of our attention. We isolate ourselves from others and the world around us. Our desire to live becomes our primary preoccupation. Exploring survival methods frequently leads to drug and alcohol abuse. Drugs and alcohol can cause a person to misinterpret information and actions, leading to irritability and anger. It can also bring up buried emotions and memories, resulting in an uncomfortable scenario and, in some cases, strong fury. When under the influence of drugs or alcohol, a person will say and do things without considering how they may affect others. Anger, like narcotics and alcohol, can impair a person's ability to think logically and rationally. To "have a horrible day," we don't need to be high on drugs or drink, nor do we need to be stressed or anxious. Recent irritations are minor irritants that accumulate during the day and reduce our tolerance for frustration. Getting out of the house, hurrying to work to reach on time just to see a punctured wheel of your car are examples of such annoyances. These irritations will mount up until you can no longer tolerate any more frustrations at the end of the day. The next minor irritant might quickly escalate into fullfledged rage. When a person feels furious, they usually exhibit some type of physiological symptom, such as those listed above. The following are some more common indications of rage:

A significant rise in the rate of breathing Muscle tenseness that isn't conscious, notably in the face and neck

Hot and cold flushes, sweating and shaking hands

An increase in blood pressure causes the face to turn pale or red, and veins prominent.

Goosebumps

Anger isn't necessarily a bad thing. It's one of the simplest protective mechanisms we have. Anger has both beneficial and negative consequences. It protects and motivates us against being abused or exploited. For example, after a long period of spending time in a toxic relationship, an individuals' rage builds up to the point that he or she feels compelled to leave in order to shut off further abuse. However, if they use this anger to control others and make others fear them, they become the abuser or bully, as previously described.

\section{Need of the Study}

Anger has been subjected to being a root cause for a plethora of emotions - mostly negative. Mental health professionals around the world from psychologists to teachers have expressed their concern regarding anger, its expression and its management. This study provides a window to the ground reality of anger from a singular perspective.

\section{Objective}

The objective of the study is to understand anger in the simplest way and how humans express it in different ways regardless of age, gender, education and occupation differences.

\section{Methodology}

The sample was selected on the basis of a simple random sampling method. This consists of 182 people between the age group of 18-72 years. The gender, age, qualification, occupation, class and religion was asked.

\section{Tools Used}

The Novaco Anger Scale (NAS) is a self-report questionnaire with Cognitive, Arousal, and Behavioural subscales that constitute a 48 -item NAS Total score. It has a separate 12-item Anger Regulation subscale. This questionnaire is designed to index a person's disposition for 
anger, which is a risk factor for violence and a dynamic variable amenable to treatment. The NAS was developed in conjunction with the violence risk project of the MacArthur Foundation Research Network on Mental Health and Law.

In its underlying variant, the NAS Total had an interior unwavering quality (alpha) of .95 and a 2-week test-retest dependability of .84 in investigations with mental patients in the California State medical clinics. A free review with male wrongdoers in Canada tracked down NAS Total alpha equivalent to .95 and testretest unwavering quality to be .89 for a 4 -week stretch. In the MacArthur Violence Risk Project implying 1,101 common responsibility mental patients, who were given the NAS while in medical clinic, the alpha for NAS Total was .94. For the WPS normalisation test, it was .94. In different investigations, the NAS Total alpha was .97 for Vietnam battle veterans, .95 for criminological patients in Scotland, .92 for formative debilitated measurable patients in England, .94 for rough detainees in Sweden, and .90 to .93 for students in California, Australia, and Sweden.

Provocation Inventory $(P I)$, related to its distribution by Western Psychological Services (WPS). The NAS subscales relate to outrage attitude areas, as connected to an ecological setting. The PI surveys self-revealed outrage force because of inciting circumstances. The two instruments were created and approved for use with intellectually cluttered and ordinary populaces.

\section{Research Design}

The exploratory research design is used in this study to understand anger and its human expression.

\section{Procedure}

The data was collected through Google forms. The participants of the research were informed about the aim of the research. Clear instruction was given to participants about each scale. Confidentiality about the participant's information will be respected. Information will be strictly used for research purposes only.

\section{Statistics}

The descriptive statistics will be used to study demographic detail of the sample.

\section{Result}

Analysis of the data was done using SPSS software. In our sample study, we obtained 182 responses.

Table 1: Showing the descriptive statistics

\begin{tabular}{|l|l|l|}
\hline Category & Parameter & Result \\
\hline Low & $0-45$ & 69 \\
\hline Mild & $46-55$ & 44 \\
\hline Moderate & $56-75$ & 54 \\
\hline High & $76-85$ & 14 \\
\hline Very high & $86-100$ & 1 \\
\hline \multicolumn{3}{|l|}{} \\
In the above table, we can see that out of these 182 responses, 69 \\
responses belong to the low category i.e. 0-45.
\end{tabular}

Out of 182 responses, 44 responses belong to the mild category i.e. $46-55.54$ responses out of 182 belong to the moderate category ranging from 56 to 75 score. 14 responses belong to the high category that extends from 76 to 85 . 1 response that was left out of 182 total responses belonged to the very high category i.e. 86-100. 
Table 2: Showing the descriptive statistics

\begin{tabular}{|l|l|l|l|l|l|}
\hline & N Statistic & $\begin{array}{l}\text { Minimum } \\
\text { Statistic }\end{array}$ & $\begin{array}{l}\text { Maximum } \\
\text { Statistic }\end{array}$ & $\begin{array}{l}\text { Mean } \\
\text { Statistic }\end{array}$ & $\begin{array}{l}\text { Standard } \\
\text { Deviation } \\
\text { Statistic }\end{array}$ \\
\hline Anger & 181 & 2.00 & 89.00 & 51.0773 & 17.01126 \\
\hline $\begin{array}{l}\text { Valid } \\
\text { (listwise) }\end{array}$ & 181 & & & & \\
\hline
\end{tabular}

In the above table, we can see that the mean and deviation of anger measured is 51.0773 and 17.01126 respectively.

\section{Diagram 1: Showing the distribution of gender}

In the above diagram, a pie chart is presented for better understanding. This pie chart displays the percentage of men and

\section{Gender}

182 responses

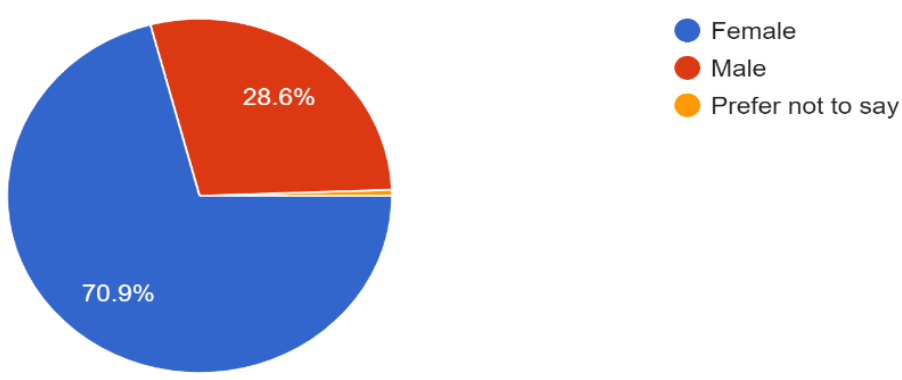

women who account for the total responses attained by the sample study for the paper

\section{Diagram 2: Showing the distribution of age across the population}

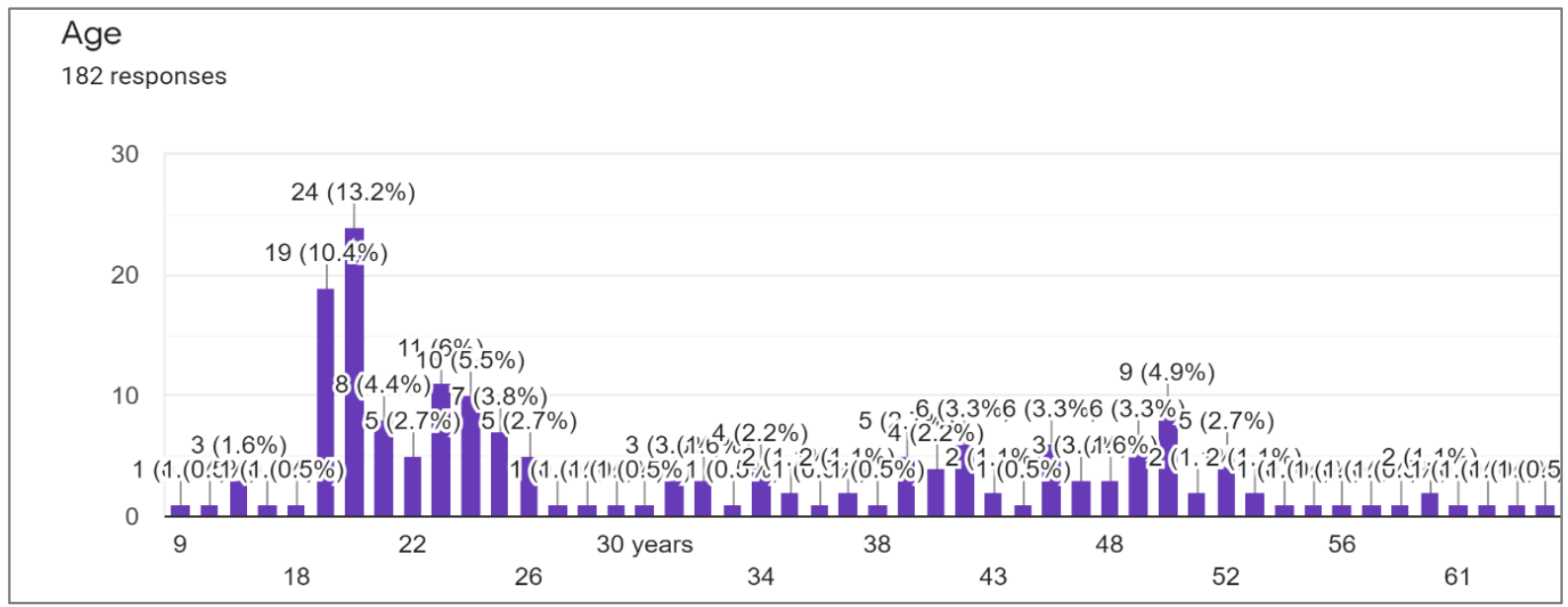

In the above diagram, a bar graph is presented displaying the varying ages of the responders. The varying ages provide us with an insight of the topic from people belonging to all age groups. 


\section{Discussion}

To judge the scoring the authors employed the Novaco Anger Scale as explained above. The said scale has been divided into sections according to its scoring to evaluate an individual's anger and irritation levels in their daily lives. The questionnaire used statements to describe a particularly trying situation that can happen to anyone anytime and gave the responders a scale ranging from 1 to 5 to mark their gauged response to the hypothetical situation. In the scale, 1 was denoted as the least amount of anger displayed, expressed or felt by the responder and 5 as the highest amount of anger. Whatever the responder marked for each question from this scale was all added and an overall score was hence achieved.

The highest score that can be achieved is 100 and the lowest being 0 .

Individuals who had their overall score between 0 to 45 were concluded as having the amount of anger or annoyance as remarkably low. They display a rather tranquil state of mind and little things do not irritate them.

Individuals who had their overall score between 46 to 55 were concluded as displaying a more peaceful disposition than an average person. People belonging to this group are patient and tend not to overreact in trying situations of life. Individuals who had their overall score between 56 to 75 had an average human being's amount of anger. The people may get irritated when in a foul mood but often keep their calm. Individuals who had their overall score between 76 to 85 often tend to react in an angry manner to daily hassles. They are a tad bit more irritable than an average human being. They tend to be quick to react and may be impulsive in nature.

Individuals who had their overall score between 86 to 100 display a very high amount of anger which is likely to be unwarranted in life's petty situations. Their anger is intense and they are likely to hold grudges for a long time. People coming in this range tend to get frequent tension headaches and elevated blood pressure. Only a few percent of the adult population belong to this range. One enormous review distributed in Circulation in 2000 observed that among 12,986 moderately aged AfricanAmerican and white people, the individuals who evaluated high in attributes like resentment
- - yet had ordinary circulatory strain - - were more inclined to coronary corridor sickness (CAD) or cardiovascular failure. Indeed, the angriest individuals confronted generally double the gamble of $\mathrm{CAD}$ and very nearly multiple times the gamble of coronary episode contrasted with subjects with the most reduced degrees of outrage. How does antagonism damage the heart? Like different types of pressure, outrage sets off a flood in adrenaline, the pressure chemical that helps the circulatory strain and heartbeat rate, expanding the heart's responsibility and increasing its requirement for oxygen. Adrenaline can likewise incite strange heart rhythms, including possibly deadly ventricular arrhythmias. What's more, the chemical enacts platelets, the small platelets that trigger blood clusters that can impede corridors restricted by the cholesterol-loaded plaques of atherosclerosis. Significant degrees of outrage might actually incite fit in a coronary vein, which brings about the extra restriction of a somewhat hindered vein.

\section{Limitations}

The result was collected via Google forms which also means that chances of biassed and socially desirable answers were high. This research was done on a qualitative basis and so in depth interviews were not conducted. The descriptive analysis pertains to mean, median and mode and standard deviation. Correlational research was not conducted.

\section{Conclusion}

Our study had an intriguing result. Majority of the responses rank low in their anger disposition while in stark contrast only one response ranked very high. Anger has been known to be detrimental to health and interpersonal relationships. In a cross cultural study of anger and stress done by Naeimeh Moheb and Usha Ram it was found that social norms highly emphasised on controlling emotions that might be socially offensive in India. This resulted in an additional pressure on the people. Barrett and Mills directed a review to analyse the emotional well-being relates to outrage in everyone in Australia. The outcome showed that a scope of disposition, tension, and substance use problems were viewed as freely connected with manifestations of outrage. Painuly et al. directed a review to investigate outrage assaults in burdensome and nervousness problems. The outcome showed 
that outrage assaults were related with more uneasiness and touchiness and more unfortunate personal satisfaction. The recurrence of outrage assaults had a positive connection with despondency, touchiness, and hostility. With our survey, we have established that most people feel angry but only once in a while.

\section{References:}

1. Anger | Psychology Today. (n.d.). Www.psychologytoday.com. https://www.psychologytoday.com/int1/basics/anger

2. Novaco, R. W. (2016, January 1). Chapter 35 - Anger (G. Fink, Ed.). ScienceDirect; Academic Press. https://www.sciencedirect.com/science/article/pii/B9 780128009512000352

3. Tavris, C. (1989). Anger: The Misunderstood Emotion. In Google Books. Simon and Schuster. https://www.google.co.in/books/edition/Anger/9NMs Tpe1bAUC?hl=en\&gbpv=1\&dq=anger\&pg=PA70\& printsec=frontcover

4. Hupka, R. B., Zaleski, Z., Otto, J., Reidl, L., \& Tarabrina, N. V. (1997). The Colors of Anger, Envy, Fear, and Jealousy. Journal of Cross-Cultural Psychology, 28(2), 156-171. https://doi.org/10.1177/0022022197282002

5. V. Dolzhenko, A., V. Kalinin, D., \& A. Kalinina, S. (2013). A New Synthesis of Amino Substituted Azolo[1,3,5]triazines via Reaction of N1,N1Dimethyl-N2-azolylformamidines with Cyanamide. HETEROCYCLES, 87(1), https://doi.org/10.3987/com-12-12601

6. Renwick, S. J., Black, L., Ramm, M., \& Novaco, R. W. (1997). Anger treatment with forensic hospital patients. Legal and Criminological Psychology, 2(1),
103-116. 8333.1997.tb00336.x

7. Eisler, R. M., Skidmore, J. R., \& Ward, C. H. (1988). Masculine Gender-Role Stress: Predictor of Anger, Anxiety, and Health-Risk Behaviors. Journal of Personality Assessment, 52(1), 133-141. https://doi.org/10.1207/s15327752jpa5201 12

8. Cultural differences in emotion: differences in emotional arousal level between the East and the West (nih.gov)

9. Storebeck J., Clore G.L. Affective arousal as information: how affective arousal influences judgments, learning, and memory. Soc Personal Psychol Compass. 2008;2:1824-1843. [PMC free article] [PubMed] [Google Scholar]

10. Shott S. Emotion and social life: a symbolic interactionist analysis. Am J Sociol. 1979;84:13171334. [Google Scholar]

11. Tsai J.L., Levenson R.W., McCoy K. Cultural and temperamental variation in emotional response. Emotion. 2006;6:484-497. [PubMed] [Google Scholar]

12. Kacen J.J., Lee J.A. The influence of culture on consumer impulsive buying behavior. J Consumer Psychol. 2002;12:163-176. [Google Scholar]

13. Rein In the Rage: Anger and Heart Disease. (2007, February 13). WebMD. https://www.webmd.com/heart-disease/features/reinin-rage-anger-heart-disease

14. Harvard Health. (2006, September 1). Anger: Heartbreaking at any age. https://www.health.harvard.edu/heart-health/angerheartbreaking-at-any-age

15. Moheb, N., \& Ram, U. (2010). Cross-cultural study of stress and anger. Procedia - Social and Behavioral Sciences, $\quad 5, \quad 1765-1769$. https://doi.org/10.1016/j.sbspro.2010.07.361

16. Dhasmana P, Singh GM, Srinivasan M, Kumar S. Anger and psychological well-being: A correlational study among working adults in Uttarakhand, India. Int J Med Sci Public Health 2018;7(4):296-300 